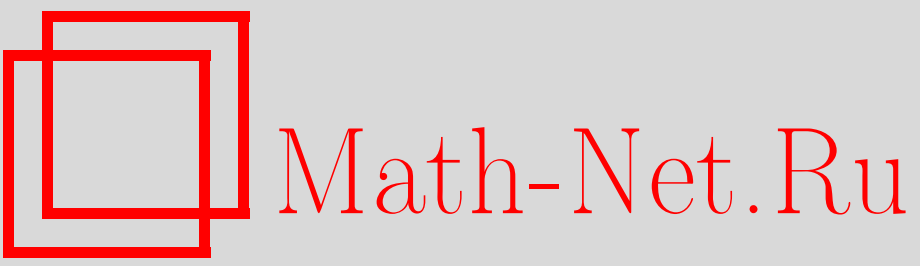

Б. Н. Четверушкин, И. А. Знаменская, А. Е. Луцкий, Я. В. Ханхасаева, Численное моделирование взаимодействия и эволюции разрывов в канале на основе компактной формы квазигазодинамических уравнений, Матем. моделирование, 2020, том 32, номер 5, 44-58

DOI: https://doi.org/10.20948/mm-2020-05-03

Использование Общероссийского математического портала Math-Net.Ru подразумевает, что вы прочитали и согласны с пользовательским соглашением

http://www.mathnet.ru/rus/agreement

Параметры загрузки:

IP: 107.22 .136 .117

26 апреля 2023 г., 18:25:46 


\title{
ЧИСЛЕННОЕ МОДЕЛИРОВАНИЕ ВЗАИМОДЕЙСТВИЯ И ЭВОЛЮЦИИ РАЗРЫВОВ В КАНАЛЕ НА ОСНОВЕ КОМПАКТНОЙ ФОРМЫ КВАЗИГАЗОДИНАМИЧЕСКИХ УРАВНЕНИЙ
}

\author{
(C) 2020 2. $\quad$ Б.Н. Четверушкин ${ }^{1}$ И.А. Знаменская ${ }^{2}$, А.Е. Луцкий \\ Я.В. Ханхасаева ${ }^{1}$
}

${ }^{1}$ Институт прикладной математики им. М.В. Келдыша РАН office@keldysh.ru, allutsky@yandex.ru, hanhyana@mail.ru

${ }^{2}$ Физический факультет МГУ им. М.В. Ломоносова znamen@phys.msu.ru

Работа выполнена при поддержке РНФ, проект 19-11-00104.

DOI: $10.20948 / \mathrm{mm}-2020-05-03$

Выполнено численное моделирование эволюции и взаимодействия разрывов течения в канале, вызванных импульсным объемным разрядом. Алгоритм построен на основе системы квазигазодинамических уравнений в компактной форме. Представлено сравнение с экспериментальными данными и результатами расчетов на основе уравнений Эйлера и Навье-Стокса.

Ключевые слова: квазигазодинамическая система уравнений, энерговложение, течение в канале, неустойчивость Рихтмайера-Мешкова.

\section{NUMERICAL SIMULATION OF INTERACTION AND EVOLUTION OF DISCONTINUITIES IN A CHANNEL BASED ON A COMPACT FORM OF QUASI-GASDYNAMIC EQUATIONS}

\author{
B.N. Chetverushkin ${ }^{1}$, I.A. Znamenskaya ${ }^{2}$, A.E. Lutsky ${ }^{1}$, Ya.V. Khankhasaeva ${ }^{1}$ \\ ${ }^{1}$ Keldysh Institute of Applied Mathematics RAS \\ ${ }^{2}$ Lomonosov Moscow State University
}

A numerical simulation of the evolution and interaction of flow discontinuities in a channel caused by a pulsed volume discharge has been performed. The algorithm is based on a system of quasi-gasdynamic equations in compact form. A comparison is made with experimental data and calculation results based on the Euler and Navier-Stokes equations.

Key words: quasi-gasdynamic system of equations, energy input, channel flow, Richtmyer-Meshkov instability. 


\section{1. Введение}

Исследование сверхзвуковых течений в каналах представляет большой теоретический и практический интерес. В последние годы повышенное внимание к этим течениям связано с разработкой высокоскоростных летательных аппаратов с прямоточными реактивными двигателями (ПВРД). Важной частью ПВРД является воздухозаборник тракта двигателя, в котором происходит торможение вязкого сверхзвукового потока, что представляет собой сложную комбинацию взаимодействия ударных волн с развивающимися турбулентными пограничными слоями, приводящую к формированию псевдоскачка [1]. Длина псевдоскачка зависит от числа Маха набегающего потока $M_{\infty}$, толщины пограничного слоя, числа Рейнольдса $\operatorname{Re}_{\infty}$ и других параметров.

При этом структура течения является весьма чувствительной к параметрам потока как на входе, так и на выходе из воздухозаборника [2]. В некоторых случаях может наблюдаться эффект запирания потока («незапуска» воздухозаборника), когда система скачков выбивается из внутреннего канала и располагается перед входом в воздухозаборник, что приводит к резкому снижению тяги двигателя или даже полному нарушению его работы. Для предотвращения запирания потока могут быть применены различные средства. Один из подходов состоит в локализованном подводе энергии посредством электрического разряда.

Ряд результатов численно-экспериментальных исследований эволюции и взаимодействия разрывов течения в канале, вызванных электрическим разрядом, были представлены в [3]. В этой работе численное моделирование проводилось в рамках математической модели уравнений Эйлера, что не позволило выявить влияние пограничного слоя на протекание газодинамических процессов.

В настоящей работе предпринята попытка восполнить указанные недостатки. Расчеты были проведены с использованием системы квазигазодинамических уравнений (КГД) в компактной форме [4,5]. Совершенствование диагностического комплекса установки также позволило получить ряд новых экспериментальных результатов.

\section{2. Экспериментальные исследования}

Серия экспериментов была проведена на физическом факультете МГУ им. М.В. Ломоносова на стенде УТРО-3 (рис.1) в ударной трубе сечением $24 \times 48$ мм с разрядной секцией. На схеме: 1 - осциллограф, 2 - генератор импульсов, 3 - вспышка, 4 - диафрагма, 5, 12 - собирающие линзы, 6 - поворотная призма, 7 - цифровой фотоаппарат, 8 - генератор импульсов, 9 блок запуска разряда, 10 - компьютер, 11 - высокоскоростная камера. 
В качестве рабочего газа использовался воздух, толкающего - гелий. Число Маха в рабочей камере варьировалось от 2 до 4.5. Давление перед фронтом ударной волны $S_{0}$ составляло $10^{3}-10^{4}$ Па. При инициировании в рабочей секции импульсного объемного разряда в прямоугольном канале в момент прохождения плоской ударной волны между плазменными электродами плазма мгновенно (за субмикросекундные времена) локализуется в области низкой плотности, отделенной фронтом волны. Длина разрядной секции от фронта ударной волны до конца разрядного промежутка варьировалась от 9 до 1 см.

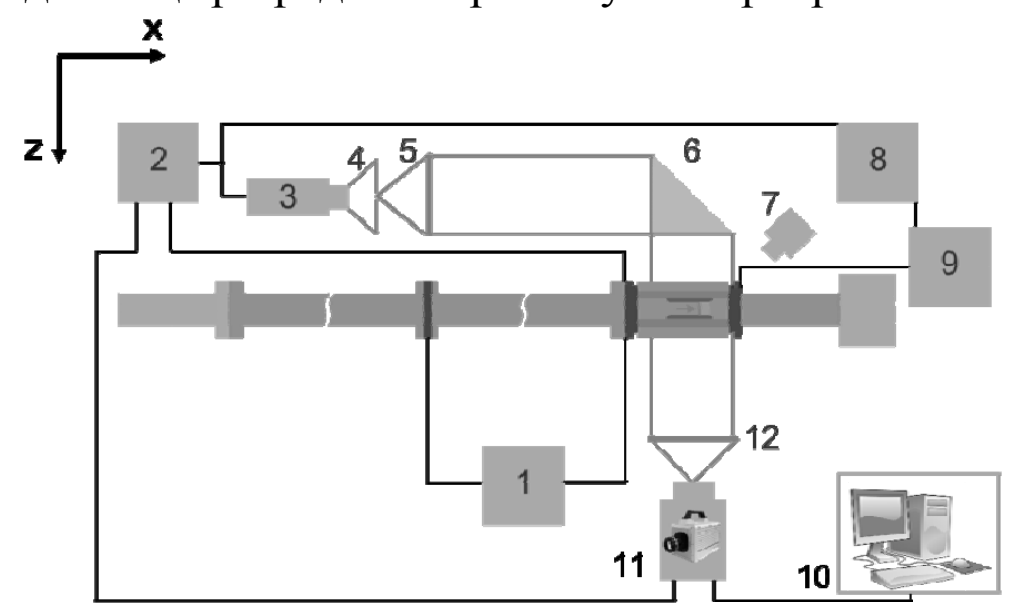

Рис.1. Схема экспериментальной установки.

После ионизации полупространства перед фронтом ударной волны в газодинамическом канале наносекундным объемным разрядом (рис.2), исходная ударная волна распадается на три поверхности разрыва - 2 ударных волны и контактную поверхность. Во всех рассматриваемых конфигурациях контактная поверхность отделяет область газа за ударной волной от области неравновесной релаксирующей плазмы - две области одинакового давления и скорости.

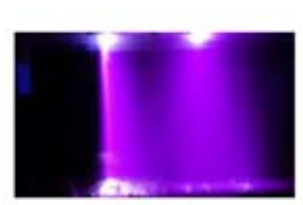

a)
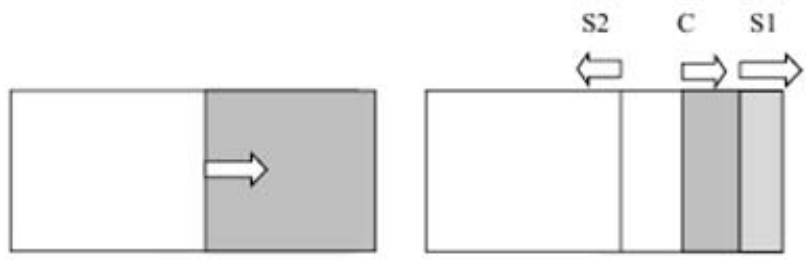

б)

Рис.2. Интегральное изображение разряда, ограниченного плоской ударной волной (а) и схема распада разрыва (б). Волна движется слева направо.

Высокоскоростная теневая съемка динамики разрывов после распада плоского разрыва проводилась на временном интервале от 1.5 до 40 мкс после разряда. Использовалась высокоскоростная камера, частота кадров составляла от 150 до 525 тыс. кадров/с. Экспозиция каждого кадра - 1 мкс. 
Регистрация динамических параметров, возникших после распада разрыва, позволяет определить, в частности, долю электрической энергии, перешедшей во внутреннюю энергию газа на стадии протекания тока разряда.

\section{3. Численный алгоритм на основе компактной формы КГД системы уравнений}

В [6] был предложен компактный вариант КГУ системы, который в двумерной постановке может быть представлен следующим образом:

$$
\begin{aligned}
& \frac{\partial Q}{\partial t}+\frac{\tau}{2} \frac{\partial^{2} Q}{\partial t^{2}}+\frac{\partial F}{\partial x}+\frac{\partial G}{\partial y}=0, \quad F=F^{i}+F^{v}, \quad G=G^{i}+G^{v} \\
& Q=\left(\begin{array}{c}
\rho \\
\rho u \\
\rho v \\
E
\end{array}\right), \quad F^{i}=\left(\begin{array}{c}
\rho\left(u-w_{1}\right) \\
\rho u\left(u-w_{1}\right)+p \\
\rho u\left(v-w_{2}\right) \\
(E+p)\left(u-w_{1}\right)
\end{array}\right), \quad F^{v}=\left(\begin{array}{c}
0 \\
-\sigma_{x x} \\
-\sigma_{x y} \\
-u \sigma_{x x}-v \sigma_{x y}-q_{x}
\end{array}\right), \\
& G^{i}=\left(\begin{array}{c}
\rho\left(v-w_{2}\right) \\
\rho v\left(u-w_{1}\right) \\
\rho v\left(v-w_{2}\right)+p \\
(E+p)\left(v-w_{2}\right)
\end{array}\right), \quad G^{v}=\left(\begin{array}{c}
0 \\
-\sigma_{x y} \\
-\sigma_{y y} \\
-u \sigma_{x y}-v \sigma_{y y}-q_{y}
\end{array}\right), \\
& w_{1}=\frac{\tau}{2 \rho}\left(\frac{\partial}{\partial x}\left(\rho u^{2}+p\right)+\frac{\partial}{\partial y}(\rho u v)\right), \quad w_{2}=\frac{\tau}{2 \rho}\left(\frac{\partial}{\partial x}(\rho u v)+\frac{\partial}{\partial y}\left(\rho v^{2}+p\right)\right) \\
& \sigma_{x x}=\frac{2}{3} \mu\left(2 \frac{\partial u}{\partial x}-\frac{\partial v}{\partial y}\right), \quad \sigma_{x y}=\sigma_{y x}=\mu\left(\frac{\partial u}{\partial y}+\frac{\partial v}{\partial x}\right), \quad \sigma_{y y}=\frac{2}{3} \mu\left(2 \frac{\partial v}{\partial y}-\frac{\partial u}{\partial x}\right) \\
& q_{x}=\kappa \frac{\partial T}{\partial x}, \quad q_{y}=\kappa \frac{\partial T}{\partial y} .
\end{aligned}
$$

Здесь $t$ - время; $x, y$ - пространственная координата; $\rho$ - плотность; $(u, v)$ - вектор скорости; $E$ - полная энергия; $p$ - давление, вычисляется по уравнению состояния совершенного газа

$$
p=(\gamma-1) \rho\left(E-\left(u^{2}+v^{2}\right) / 2\right),
$$

$\gamma$ - показатель адиабаты, для воздуха $\gamma=1.4 ;\left(q_{x}, q_{y}\right)$ - вектор теплового потока, $T$ - температура, $\kappa$ - коэффициент теплопроводности; $\sigma$ - тензор вязких напряжений в уравнениях Навье-Стокса, молекулярная вязкость $\mu$ определяется соотношением 
$\mu / \mu_{0}=\left(T / T_{0}\right)^{s}, \quad s=0.76$,

$\mu_{0}$ - молекулярная вязкость при опорной температуре $T_{0} ; \tau=\mu / p$ - характерное время между столкновениями молекул.

Величина $w_{i}$ с точностью до множителя $\tau / 2 \rho$ совпадает с пространственными производными, входящими в уравнение, описывающее изменение импульса в системе уравнений Эйлера. Таким образом, для аппроксимации пространственных производных в системе (1) можно практически без изменения использовать алгоритмы, применяемые для решения уравнений Навье-Стокса и Эйлера.

Система (1) при использовании метода конечного объема и трехслойной явной схемы принимает вид

$$
\Omega \frac{Q_{i j}^{n+1}-Q_{i j}^{n}}{\Delta t}+\Omega \frac{\tau^{*}}{2} \frac{Q_{i j}^{n+1}-2 Q_{i j}^{n}+Q_{i j}^{n-1}}{\Delta t^{2}}+\sum_{k=1}^{4}\left(F n_{x}+G n_{y}\right)_{k}=0 .
$$

Здесь суммирование ведется по всем ребрам ячейки, $F, G$ - векторы потоков, определяемые соотношениями $(1),\left(n_{x}, n_{y}\right)$ - вектор нормали, $\Omega-$ площадь ячейки.

Значения пространственных производных на ребрах ячеек находятся по шаблону на рис.3. Значения переменных в узлах сетки 3, 4 получены усреднением по соседним ячейкам. Полученные производные используются для вычисления $w_{i}$ и $\sigma$.

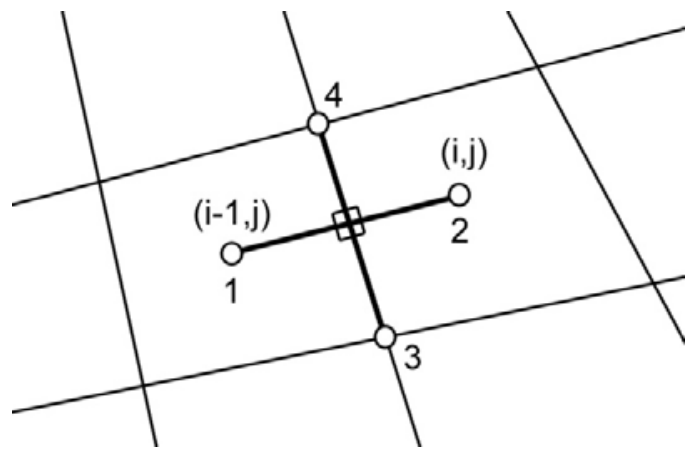

Рис.3. Шаблон для вычисления пространственных производных на ребрах ячеек.

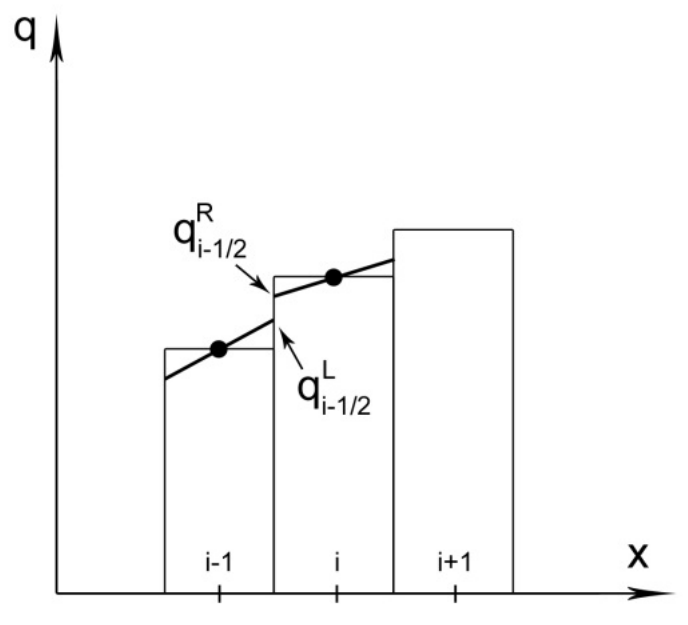

Рис.4. Кусочно-линейная реконструкция типа MUSCL.

Чтобы получить значения консервативных переменных $Q_{i j}^{n+1}$ на следующем слое по времени, необходимо по известным величинам $Q_{i j}^{n}, Q_{i j}^{n-1}$ на всех ребрах вычислить численные потоки $\left(F n_{x}+G n_{y}\right)_{k}$. Для реконструкции 
значений переменных на гранях используется схема типа MUSCL, обеспечивающая второй порядок аппроксимации по пространству (рис.4.). Газодинамические величины на ребре ячейки определяются на основе точного решения задачи Римана с начальными данными $q_{i-1 / 2}^{L}, q_{i-1 / 2}^{R}$. Аналогично определяются величины на ребрах, разделяющих ячейки $j-1, j$.

\section{4. Численное моделирование}

Постановка задачи представлена на рис.5. В начальный момент времени плоская ударная волна с числом Маха $\mathrm{M}=3$ располагается в сечении $x=0$. В эксперименте в объемную часть разряда $0<x<1.5,|y|<1.1$ (область 2 на рис.5) вкладывалось 0.83 Дж, в плазменные листы около стенок $1.1<|y|<1.2$ (область 3 на рис.5) - по 0.11 Дж. На основании ранее проведенных исследований [4] предполагалось, что в поступательные степени свободы мгновенно переходит 50\% вложенной энергии. В рассматриваемом случае вложенная энергия составляет $16.75 \%$ от полной энергии (на единицу объема) газа за фронтом падающей ударной волны.

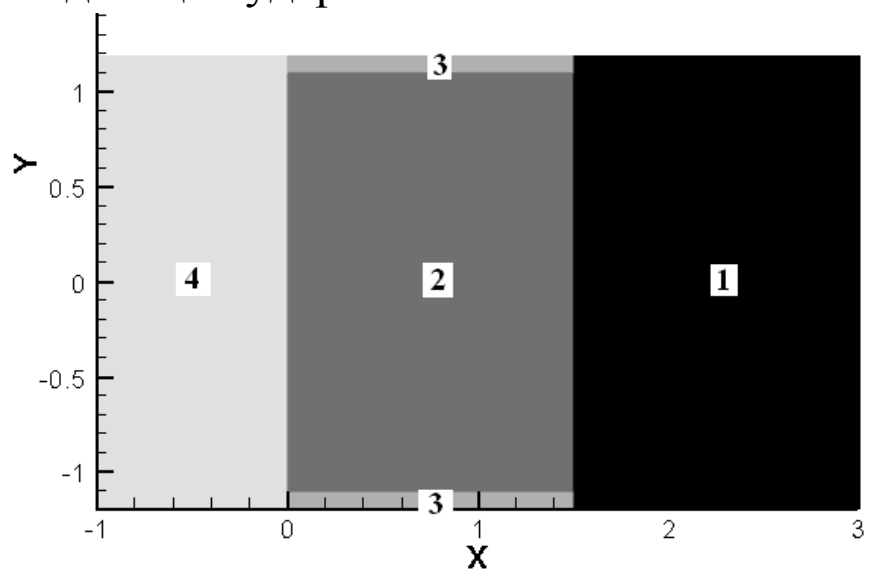

Рис.5. Начальные данные.

Равномерное по времени и пространству вложение энергии $\Delta E / V$ в постоянный поток приводит к росту давления на величину $\Delta p=(\gamma-1) \Delta E / V$ при сохранении плотности и скорости. В расчетах плотность и давление были отнесены к величинам в невозмущенном газе - область 1 на рис.5. При такой нормировке безразмерная единица времени соответствует $3.45 \times 10^{-5} \mathrm{c}$.

Таким образом, в начальный момент времени существует 5 областей с постоянными значениями газодинамических величин:

1) невозмущенный неподвижный газ перед фронтом падающей ударной волны:

$x>1.5: \rho_{1}=1, \quad u_{1}=0, \quad v_{1}=0, \quad p_{1}=1 ;$

2) объемная часть разряда: 
$0<x<1.5,|y|<1.1: \quad \rho_{2}=1, \quad u_{2}=0, \quad v_{2}=0, \quad p_{1}=6.2412$;

3) пристеночные области («плазменные листы»):

$0<x<1.5,|y|>1.1: \quad \rho_{3}=1, \quad u_{3}=0, \quad v_{3}=0, \quad p_{3}=16.2820$

4) область за фронтом падающей волны:

$x<0: \quad \rho_{4}=3.8571, \quad u_{4}=2.6294, \quad v_{4}=0, \quad p_{4}=10.3333$.

На границах этих областей в начальный момент происходит распад разрывов с образованием волн разрежения, ударных волн и тангенциальных разрывов. В последующие моменты времени течение характеризуется достаточно сложным процессом движения и взаимодействия этих вторичных разрывов.

На начальном интервале времени в областях вдали от угловых точках течение определяется одномерными задачами о распаде произвольного разрыва - в продольном и поперечном направлениях.

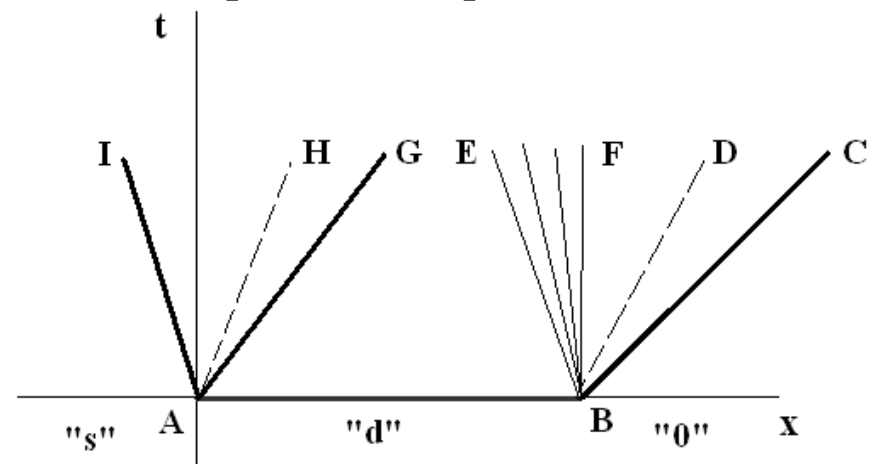

Рис.6. Начальное расположение разрывов в 1D задаче в продольном направлении.

Энергия вкладывается на отрезке АВ. В результате распада разрыва в точке А формируется ударная волна AI, тангенциальный разрыв AН, ударная волна AG. Распад разрыва в точке В дает волну разрежения BEF, тангенциальный разрыв $\mathrm{BD}$, ударную волну ВC.

В начальный момент времени давление в плазменных листах существенно превышает давление в объемной части разряда. В результате распада разрыва на границе этих областей формируется ударная волна, движущаяся к плоскости симметрии, и волна разрежения, распространяющаяся в сторону стенки. Течение в последующие моменты времени характеризуется серией отражений этих волн от границы области и их взаимодействием. Для дальнейшего важно отметить, что вблизи стенок во все последующие моменты времени сохраняется прогретый слой с температурой в среднем в 2 раза выше, чем в остальной части области. К настоящему времени достаточно хорошо изучены (см., напр., $[7,8]$ ) многие вопросы, связанные с взаимодействием ударных волн с прогретыми слоями. Основной особенностью 
такого процесса является ускоренное движение ударной волны по прогретой области, приводящее к искривлению фронта, формированию “предвестника" и вторичных разрывов.

Расчеты 2D течения проводились на сетке, содержащей $1024 \times 128$ ячеек в полуплоскости $y>0$ с учетом симметрии течения. Начальная стадия $(t=0.15)$ двумерного течения, возникающего при одновременном вложении энергии в объемную область и плазменные листы, изображена на рис.7.

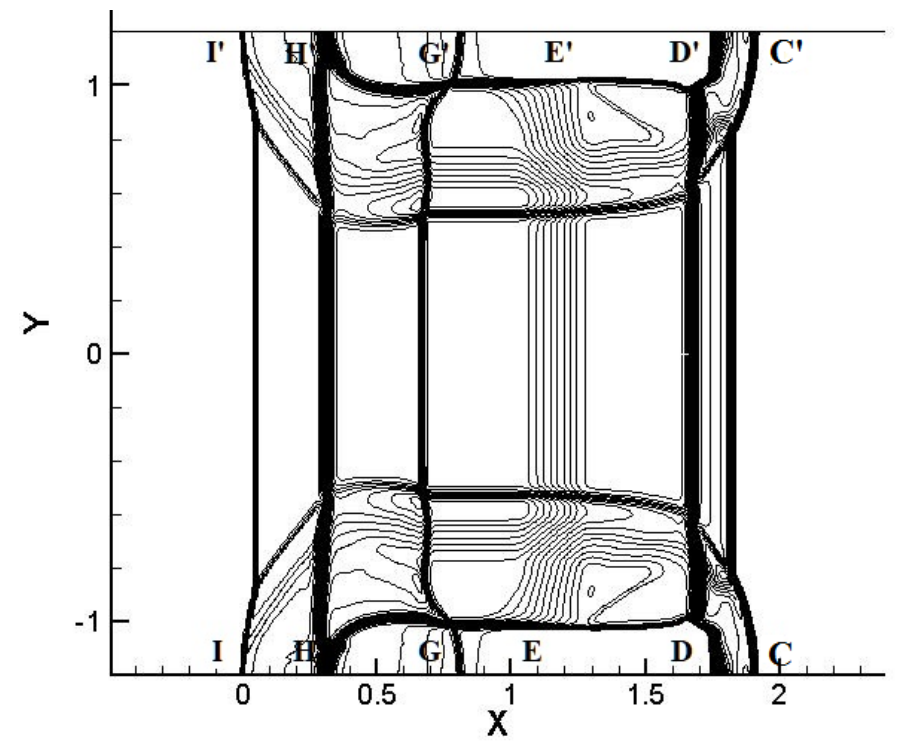

Рис.7. Структура основных разрывов. Изолинии плотности при $t=0.15$.

Вблизи плоскости симметрии ( $y=0)$ в течение начальной стадии процесса решение близко к продольному одномерному, описанному выше. Однако около стенок ударные волны I'I, C'C, G'G и тангенциальные разрывы H'H, D'D движутся с более высокими скоростями. Кроме того, на границах плазменных листов в начальный момент образуются разрывы, распространяющиеся и взаимодействующие в поперечном направлении. Линия тангенциального разрыва D’D, которая в начальный момент совпадала с правой границей области энерговыделения, достаточно быстро оказывается искривленной в силу ускоренного движения вблизи стенок. Эта стадия течения изображена на рис.8а, б.

В некоторый момент времени ударная волна $G^{\prime} G$ (также искривленная) догоняет контактный разрыв D'D. В результате взаимодействия этих разрывов образуются две ударные волны: отраженная и проходящая. Амплитуда возмущений тангенциального разрыва D’D интенсивно нарастает, рис.8в-e.

Такие свойства течения позволяют говорить о своеобразном проявлении неустойчивости Рихтмайера-Мешкова, которая по современным представлениям [7-11] характеризуется рядом следующих стадий. 
1) Линейная стадия. Рост амплитуды возмущений со временем происходит линейно со скоростью, пропорциональной частоте, амплитуде начального возмущения и числу Атвуда.

2) Нелинейная стадия. Начало формирования пузырей легкого газа, проникающих в тяжелый, и струй тяжелого, проникающих в легкий. Замедление скорости роста пузырей. Постоянная или ускоренная скорость роста струй. Начало формирования грибовидных структур на концах струй.

3) Переходная стадия. Дальнейшее развитие грибовидных структур и начало их слияния. Возникновение неустойчивости на границах струй.

4) Турбулентная стадия. Разрушение упорядоченной структуры из пузырей и струй. Образование слоя турбулентного перемешивания и рост его толщины.

В полученных численных данных достаточно отчетливо проявляются стадии 2, 3. Они представлены последовательно на рис.8в-е.

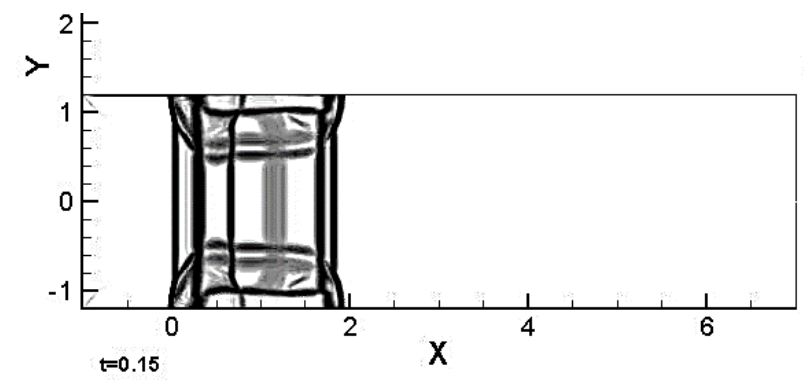

a)

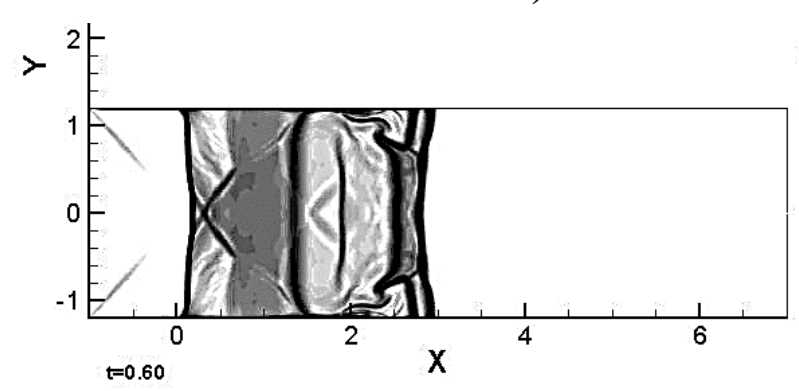

B)

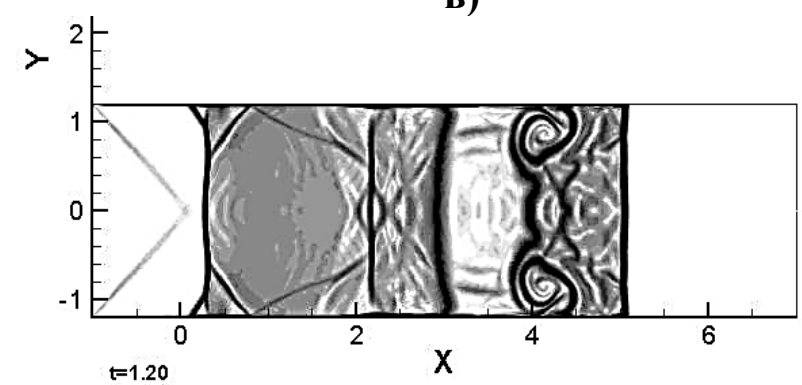

д)

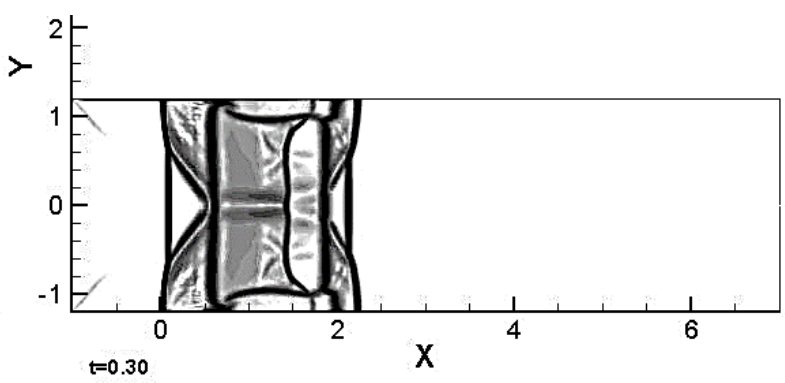

б)

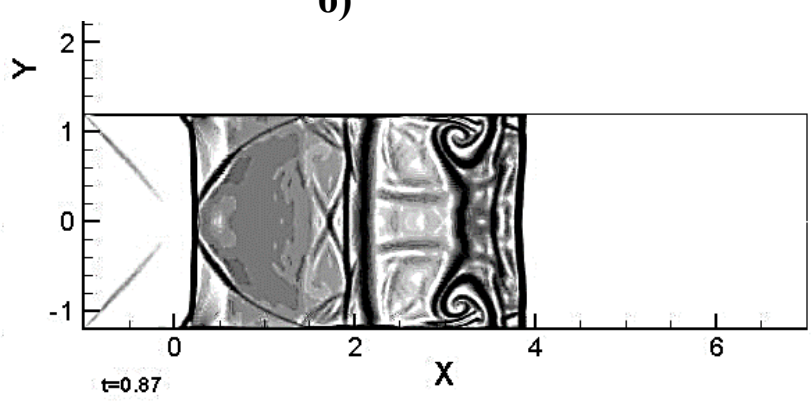

г)

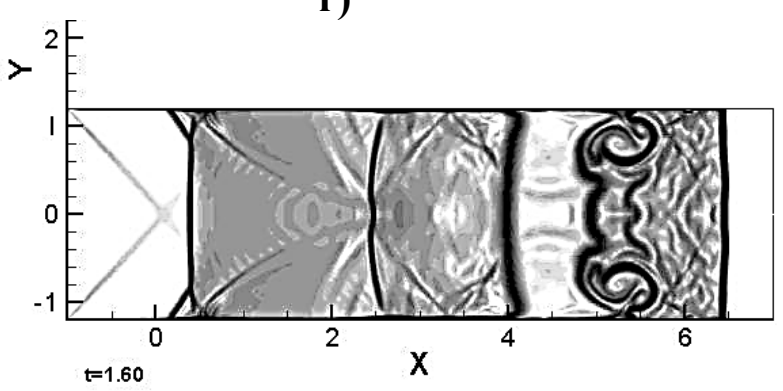

e)

Рис.8. Движение и эволюция основных разрывов. Модуль градиента плотности в последовательные моменты времени. 
Следует отметить существенные отличия исследуемого течения от классического варианта неустойчивости Рихтмайера-Мешкова, когда рассматривается падение плоской ударной волны на тангенциальный разрыв с гармоническими возмущениями. Начальное (перед приходом ударной волны) возмущение тангенциального разрыва имеет форму сглаженной ступеньки. Это возмущение формируется с самого начала процесса за счет более высокой скорости тангенциального разрыва около стенки. По той же причине падающая ударная волна является не плоской, а искривленной, также близкой по форме к ступенчатой. Дополнительное возмущение тангенциального разрыва происходит под действием поперечной ударной волны, распространяющейся от границы плазменного листа. Изучение турбулентной стадии требует проведения расчетов в 3D постановке.

Отметим, что аналогичные явления были получены также в [12]. Ряд интересных результатов по исследованию неустойчивости РихтмайераМешкова содержится в [13].

Сопоставление с результатами ранее выполненных исследований показывает, что основные закономерности движения и взаимодействия разрывов в рассматриваемом течении имеют невязкую природу. Однако использование КГД-модели позволяет выявить ряд новых деталей.

Влияние вязкости проявляется, в первую очередь, в областях около стенок канала. Для всех моментов времени, представленных на рис.8, видно формирование возмущений, вызванных развитием пограничного слоя на стенках канала.
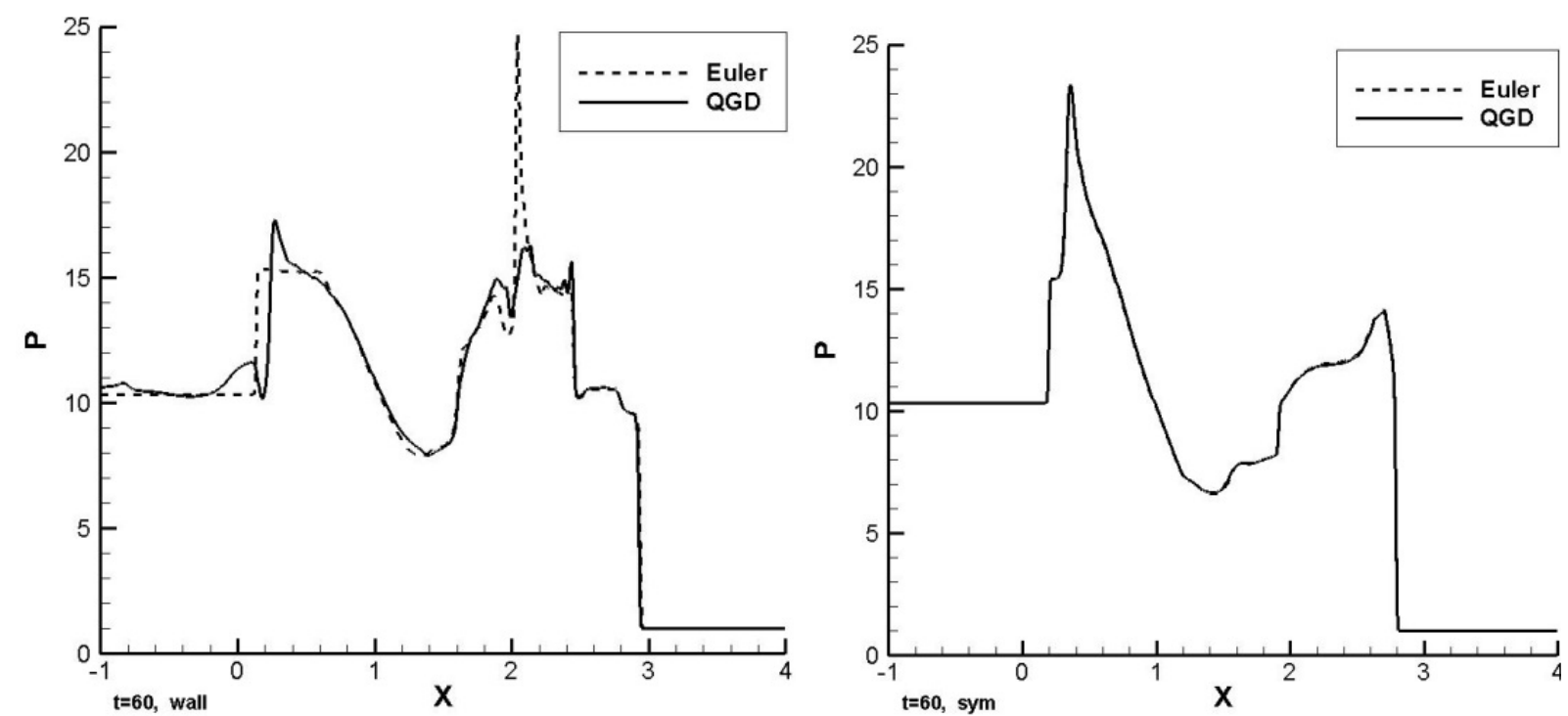

Рис.9. Распределение давления на стенке канала слева и в плоскости симметрии справа. Сплошные линии - решение КГД-системы, пунктир - уравнений Эйлера, $t=0.6$. 
На рис.9 представлено распределение давления на стенке канала и в плоскости симметрии в момент времени $t=0.6$ для решений уравнений Эйлера и КГД-системы. Наблюдаемые различия обусловлены, главным образом, взаимодействием разрывов с пограничным слоем на стенках канала. В решении уравнений Эйлера это явление, естественно, не воспроизводится.

Рост давления в окрестности точки $x=0$ связан с формированием отрыва пограничного слоя под действием ударной волны II' (рис.10). С другой стороны, наличие пограничного слоя ослабляет воздействие ударной волны $\mathrm{G}^{\prime} \mathrm{G}$ в окрестности точки $x=2$. В плоскости симметрии решения практически не различаются.

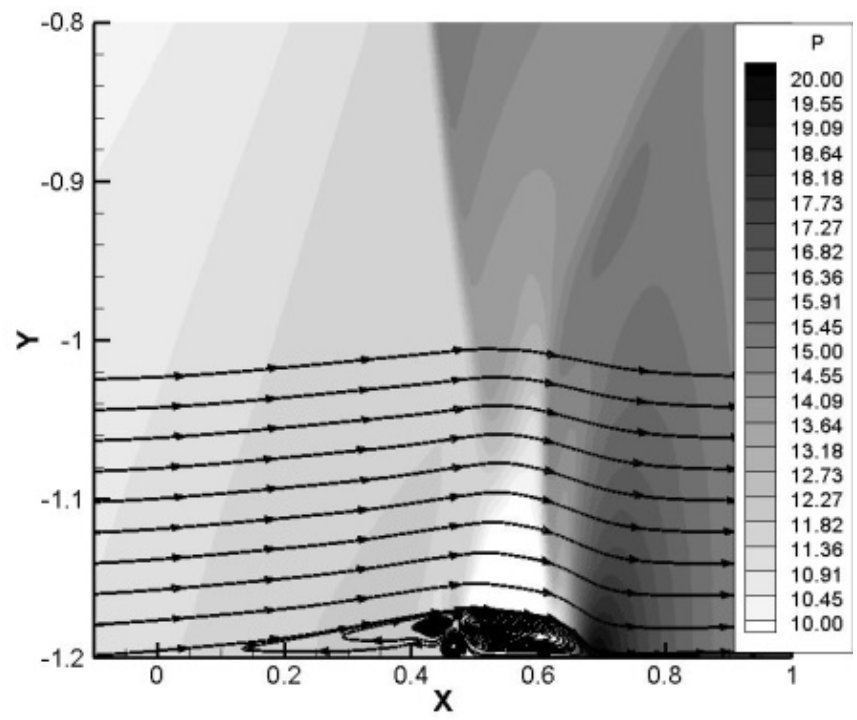

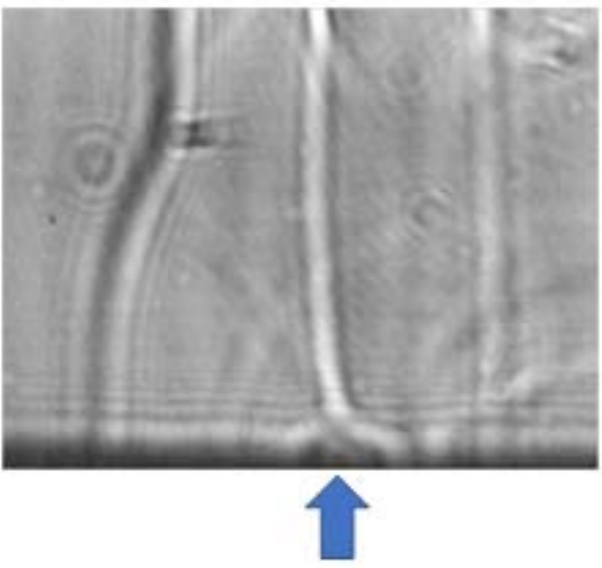

Область отрыва

Рис.10. Отрыв пограничного слоя, вызванный ударной волной II’.
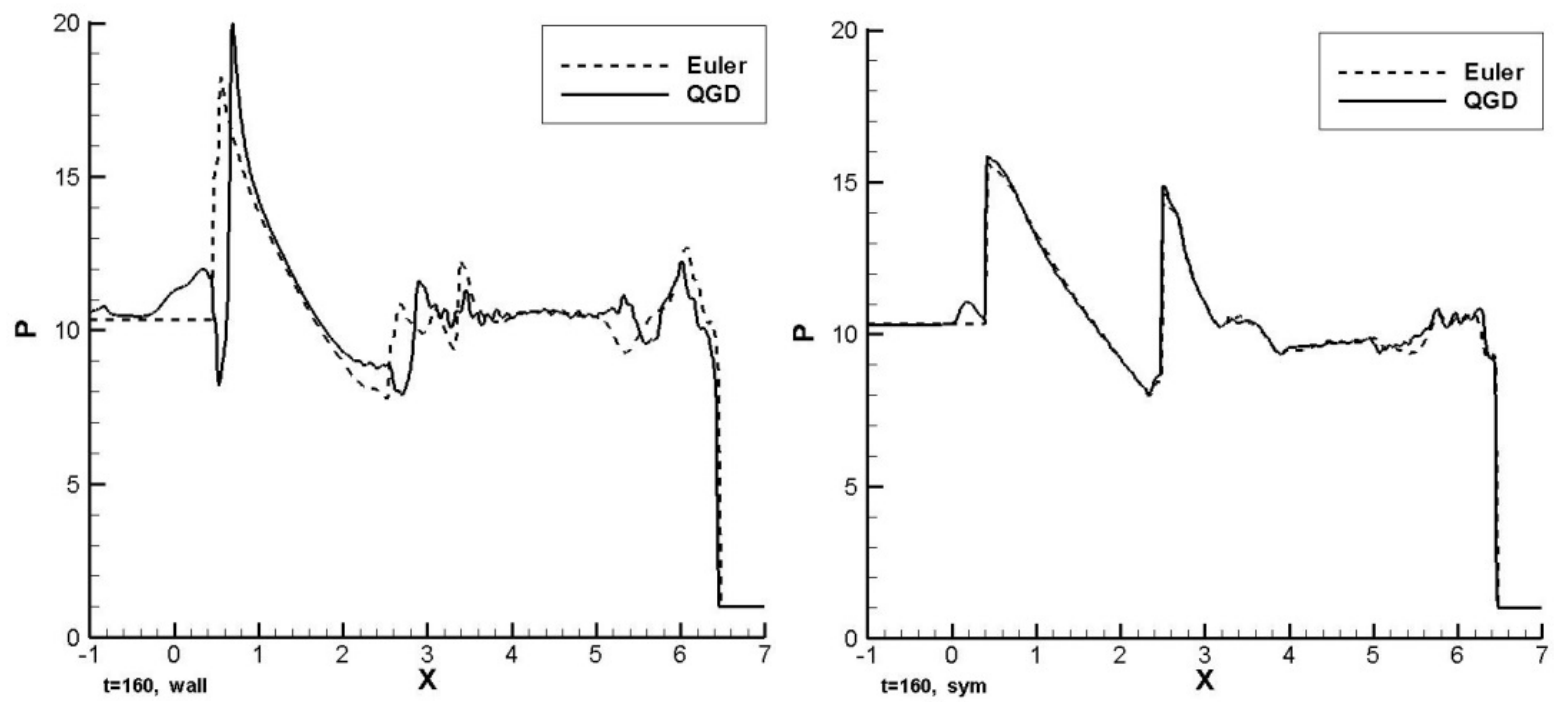

Рис.11. Распределение давления на стенке канала слева и в плоскости симметрии справа. Сплошные линии - решение КГД-системы, пунктир - уравнений Эйлера, $t=1.6$. 
К моменту времени $t=1.6$ влияние возмущений пограничного слоя распространяется на всю область течения. На рис.11 $(t=1.6)$ видны некоторые различия в распределении давления и в плоскости симметрии.

\section{5. Верификация и валидация}

С целью исследования сходимости КГД- алгоритма проведены расчеты на сетках: 1 - 512×64, 2 - 1024×128, 3 - 2048×256.

Результаты представлены на рис.12.

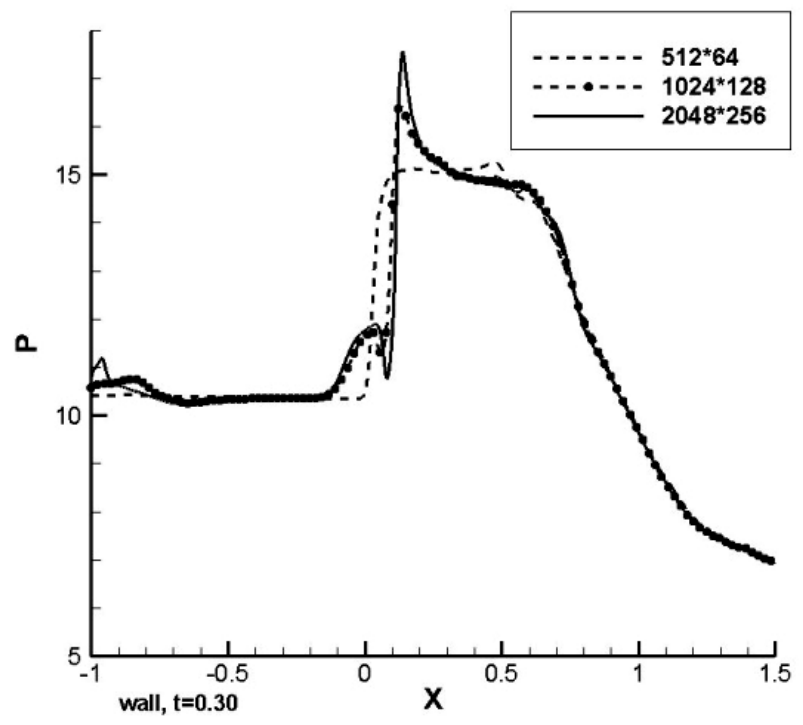

a)

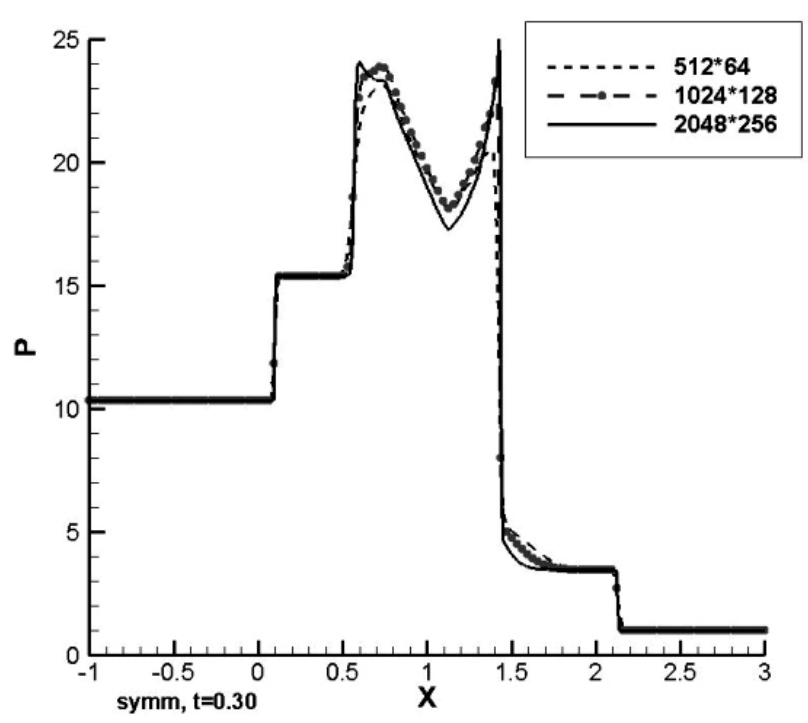

б)

Рис.12. Распределение давления: а) на нижней стенке канала, б) в плоскости симметрии.

Как видно из представленных результатов, сетка 1 является слишком грубой для воспроизведения пограничного слоя и его отрыва перед ударной волной I'I в окрестности точки $x=0$. Данные, полученные на сетках 2,3 , весьма близки за исключением максимума давления за фронтом ударной волны I'I. В плоскости симметрии распределения давления на всех 3 сетках достаточно близки.

Ранее на рис.9, 11 было представлено сравнение результатов по модели уравнений Эйлера и КГД-модели. Как отмечалось, основные отличия связаны с отсутствием пограничного слоя в модели уравнений Эйлера.

Представляет интерес также сравнение результатов, полученных в рамках модели уравнений Навье-Стокса и КГД-системы - рис.13.

В целом, результаты по двум моделям весьма близки. Для объяснения наблюдаемых различий напомним, что в представленных расчетах параметр $\tau$ выбирался локально из условия $\tau=\mu / p$. Эта величина значительно меньше, чем в классических КГД-алгоритмах, в которых конвективные производные 
аппроксимируются центральными разностями. Однако и в рассматриваемом в настоящей работе подходе коэффициенты при вторых производных (вязкость) оказываются выше, чем в стандартных уравнениях Навье-Стокса, что и определяет несколько большую диссипацию.

Сравнение результатов расчетов с теневыми фотографиями, полученными в эксперименте (рис.14), показывает, что используемая математическая модель (система КГД уравнений в компактной форме) и численный алгоритм правильно воспроизводят основные разрывы течения, их эволюцию и динамику. В частности, как в эксперименте, так и в расчете наблюдается развитие неустойчивости контактного разрыва.

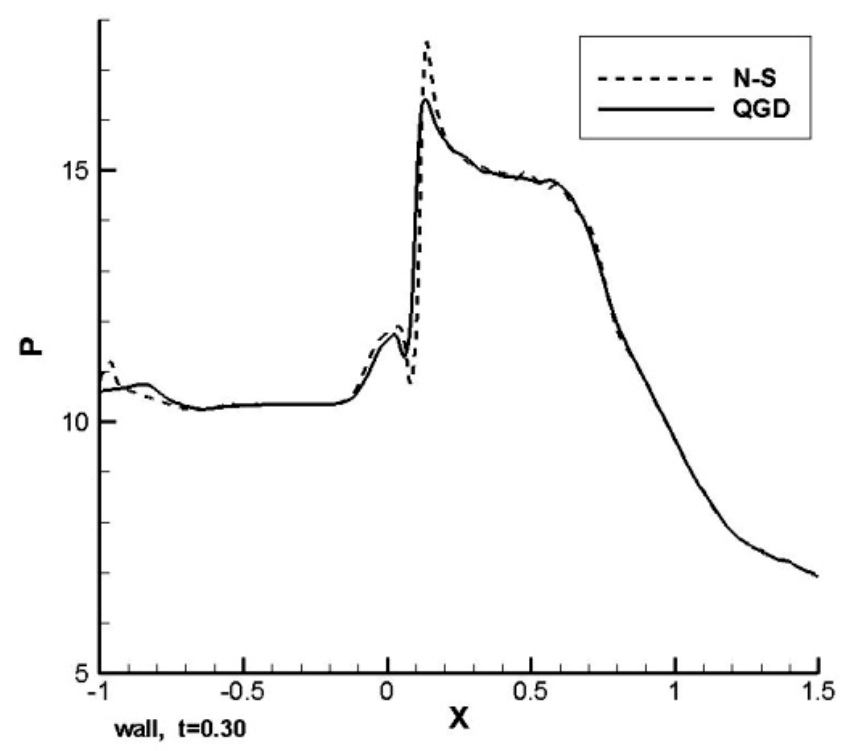

Рис.13. Распределение давления на стенке канала - результаты по моделям Навье-Стокса и КГД-системы.
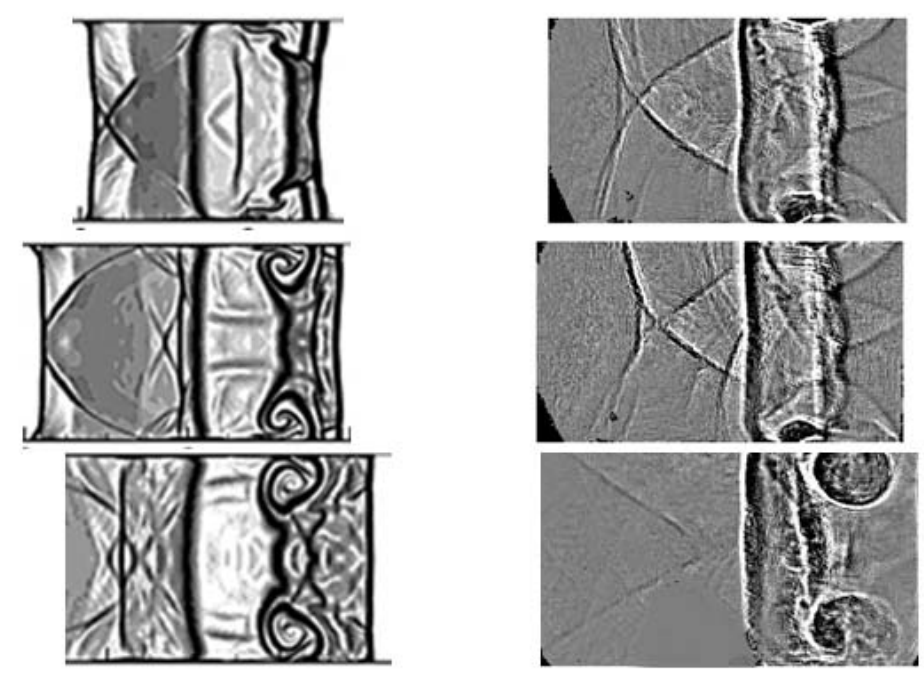

Рис.14. Модуль градиента плотности (слева) и теневые фотографии (справа) на моменты времени $t=0.6,0.87,1.2$. 


\section{6. Заключение}

Результаты численных и экспериментальных исследований эволюции и взаимодействия разрывов течения в канале, вызванных электрическим разрядом, свидетельствуют о развитии в канале неустойчивости контактного разрыва типа Рихтмайера-Мешкова.

Численные исследования на основе системы КГД-уравнений свидетельствуют о возможности отрыва пограничного слоя на стенках канала, что подтверждается и в эксперименте.

Сопоставление результатов расчетов с использованием различных математических моделей с экспериментальными данными показывают адекватность математической модели КГД-уравнений в компактной форме.

\section{СПИСОК ЛИТЕРАТУРЫ}

1. О.В. Гуськов, В.И. Копченов, И.И. Липатов, В.Н. Острась, В.П. Старухин. Процессы торможения сверхзвуковых течений в каналах. - М.: Физмалит, 2008;

O.V. Gus'kov, V.I. Kopchenov, I.I. Lipatov, V.N. Ostras', V.P. Starukhin. Processy tormozheniya sverkhzvukovykh techenii v kanalakh. - M.: Fizmatlit, 2008.

2. В.А. Забайкин. Управление псевдоскачком нестационарным воздействием // Физикохимическая кинетика в газовой динамике, 2011, т.12;

V.A. Zabaykin. Control of pseudo shock by non-stationary effect // Physical-Chemical Kinetics in Gas Dynamics, 2011, v.12. URL: http://chemphys.edu.ru/issues/2011-12/articles/353/

3. I.A. Znamenskaya, D.A. Koroteev and A.E. Lutsky. Discontinuity breakdown on shock wave interaction // Physics of Fluids, 2008, v.20, p.056101.

4. B.N. Chetverushkin. Kinetic Schemes and Quasi-Gas Dynamic System of Equations. CIMNE. - Barcelona: 2008, 298 p.

5. A.E. Lutskii, B.N. Chetverushkin. Compact Version of the Quasi-Gasdynamic System for Modeling a Viscous Compressible Gas // Differential Equations, 2019, 55(4), p.575-580.

6. Б.Н. Четверушкин, А.В. Савельев, В.И. Савельев. Квазигазодинамическая модель для описания магнитогазодинамических явлений // ЖВМиМФ, 2018, т.58, №8, c.189-199; B.N. Chetverushkin, A.V. Saveliev, V.I. Saveliev. A Quasi-Gasdynamic Model for the Description of Magnetogasdynamic Phenomena // Computational Mathematics and Mathematical Physics, 2018, v.58, Issue 8, p.1384-1394.

7. В.И. Артемьев, В.И. Бергельсон, А.А. Калмыков, И.В. Немчинов, Т.И. Орлова, В.А. Рьбаков, В.А. Смирнов, В.М. Хазинс. Развитие предвестника при взаимодействии ударной волны со слоем пониженной плотности // Изв. Ан СССР, МЖГ, 1988, №2, с.158-163;

Англ. пер.: V.I. Artem'ev, V.N. Bergel'son, A.A. Kalmykov, I.V. Nemchinov, T.I. Orlova, V.A. Rybakov, V.A. Smirnov, V.M. Khazins. Development of a forerunner in interaction of a shock wave with a layer of reduced pressure // Fluid Dynamics, 1988, v.23, Issue 2, p.290-295.

8. E. Koroteeva, I. Znamenskaya, D. Orlov, N. Sysoev. Shock wave interaction with a thermal layer produced by a plasma sheet actuator // J. of Physics D - Applied Physics, 2017, v.50, Issue 8, 085204 . 
9. R.D. Richtmyer. Taylor instability in shock acceleration of compressible fluids // Comm. Pure. Appl.Math., 1960, v.13, p.297-319.

10. E.E. Мешков. Неустойчивость границы раздела двух газов, ускоряемых ударной волной // Изв. АН СССР. МЖГ, 1969, № 5, с.151-157;

Англ. пер.: E.E. Meshkov. Instability of the interface of two gases accelerated by a shock wave // Fluid Dynamics, 1969, v.4, Issue 5, p.101-104.

11. И.Г. Лебо, В.Ф. Тишкин. Исследование гидродинамической неустойчивости в задачах лазерного термоядерного синтеза. - М.: Физматлит, 2006;

I.G. Lebo, V.F. Tishkin. Issledovanie gidrodinamicheskoi neustoichivosti v zadachakh lazernogo termoyadernogo sinteza. - M.: Fizmatlit, 2006.

12. П.Ю. Георгиевский, В.А. Левин, О.Г. Сутьрин. Двумерные автомодельные течения, порожденные взаимодействием скачка уплотнения с областями газа пониженной плотности // Механика жидкости и газа, 2010, № 2, с.126-134;

Англ. пер.: P.Yu. Georgievskii, V.A. Levin, O.G. Sutyrin. Two-dimensional self-similar flows generated by the interaction between a shock and low-density gas regions // Fluid Dynamics, 2010, v.45, Issue 2, p.281-288.

13. Ben Guan, Zhigang Zhai, Ting Si, Xiyun Lu, and Xisheng Luo. Manipulation of three-dimensional Richtmyer-Meshkov instability by initial interfacial principal curvatures // Physics of Fluids, 2017, v.29, 032106.

Поступила в редакцию 21.10.2019

После доработки 21.10.2019

Принята к публикации 25.11.2019 